\title{
Reducing co-administration of clopidogrel and proton pump inhibitors in acute coronary syndromes
}

\author{
${ }^{1}$ V Vassiliou, ${ }^{2}$ A Silva, ${ }^{3} \mathrm{~F}$ Kavvoura, ${ }^{4} \mathrm{R}$ Schofield, ${ }^{5} \mathrm{DB}$ Rowlands \\ ${ }^{1}$ Cardiology SpR, Papworth Hospital, Cambridge; ${ }^{2}$ ST1 Neurosurgery, University Hospital Coventry \& Warwickshire NHS Trust, Coventry; \\ ${ }^{3}$ Academic Clinical Lecturer in Diabetes \& Endocrinology, Oxford University, Oxford; ${ }^{4}$ Cardiology SpR, Addenbrooke's Hospital, Cambridge; \\ ${ }^{5}$ Cardiology Consultant, Peterborough City Hospital, Peterborough, UK
}

\begin{abstract}
Background: Recent concerns about clopidogrel and proton pump inhibitor (PPI) co-administration in patients following an acute coronary syndrome (ACS) led us to investigate our practice. Guidance recommends that omeprazole and esomeprazole should not be co-administered with clopidogrel as they can decrease its efficacy. Pantoprazole and lansoprazole are safer for use in these cases.

Methods: Patients discharged between August 2008 and July 2009 with a diagnosis of ACS, on both clopidogrel and any PPI, were identified using the pharmacy database. Their notes were retrospectively reviewed, the indication for a PPI was checked and whether an appropriate one had been prescribed was assessed. As a result of our findings a simple algorithm was introduced to set out guidance on appropriate use and junior doctors were informed of the new procedure. We then performed another audit of patients discharged between August 2009 and February 2010 to ensure that the guidelines were being followed. Results: During the first phase of the audit 43 out of 91 patients (47.5\%) received a PPI with clopidogrel. Following our intervention, re-auditing confirmed that clopidogrel and PPI co-administration had decreased significantly to 27 out of 101 patients $(26.7 \%)(p=0.018)$.

Conclusions: Through a simple intervention with an algorithm and education of junior doctors we have shown that significant improvement and adherence to guidance can be achieved.
\end{abstract}

KEYWORDS Clopidogrel, proton pump inhibitors, omeprazole, interaction, P450 enzymes, acute coronary syndrome

DECLARATION OF INTERESTS No conflicts of interest declared.
Correspondence to V Vassiliou, 3 Ventress Farm Court,

Cambridge CBI 8HD, UK

tel. +44 (0) I22 34I 4797 e-mail vassiliou@doctors.org.uk

\section{INTRODUCTION}

In patients with acute coronary syndromes (ACS), there is good evidence that aspirin and clopidogrel should continue to be prescribed for one year following onset to allow maximum coronary protection and minimise major adverse coronary events.' This recommendation is currently endorsed by local and national guidance. ${ }^{2,3}$

We noted however that when patients in our hospital were started on dual antiplatelet therapy there was a tendency to also initiate the use of a proton pump inhibitor (PPI), even in the absence of gastrointestinal symptoms, in order to minimise the potential risk of gastrointestinal bleeding. This appears to be a common practice around the world; in some studies PPI co-administration with clopidogrel ranged from $42 \%{ }^{4}$ to $64 \%^{5}$ of patients with ACS and the common reason for use was in order to reduce gastrointestinal bleeds.
Recent concerns have been raised about potentially adverse reactions; the European Medicines Agency (EMEA) introduced guidelines stating thatco-administration of omeprazole and esomeprazole should be avoided in patients taking clopidogrel. Other PPI such as lansoprazole and pantoprazole were considered to be safer. ${ }^{6}$ Clopidogrel is a pro-drug that must be metabolised by the body before it can become bioactive. The hepatic P450 enzymes activate it, allowing adenosine diphosphate (ADP) mediated platelet inhibition. There have been some concerns that co-administration of a PPI (in particular omeprazole or esomeprazole) can act as a strong P450 inhibitor, limiting clopidogrel conversion to its active metabolite and therefore decreasing its efficacy. Although this concern is not universally shared, ${ }^{7}$ if esomeprazole/omeprazole affect the efficacy of clopidogrel, this can lead to significant adverse events for patients following an ACS. These could include new myocardial infarction, stent thrombosis in patients with 
coronary stents and death. Therefore, although the debate is ongoing, ${ }^{8}$ the guidance from both the EMEA ${ }^{6}$ and the US Food and Drug Administration (FDA ${ }^{9}$ remains clear: use of a PPI should be avoided if not clearly required. If it is considered necessary, other PPI such as pantoprazole and lansoprazole which are considered weaker inhibitors of P450 enzymes, are currently considered safe and should be used when co-administrated with clopidogrel. Ranitidine, an $\mathrm{H}_{2}$ blocker used widely for iatrogenic dyspepsia/gastritis in the pre-PPI era, ${ }^{10}$ is also considered safe.

\section{METHODS}

We set out to retrospectively analyse our practice in Peterborough District Hospital, a large District General Hospital in Cambridgeshire, UK. We reviewed case notes from patients who were discharged between August 2008 and July 2009 following an ACS, on aspirin, clopidogrel and a PPI. A list of all eligible patients was generated from the pharmacy database. We felt that reviewing the notes of a third of the cases provided substantial evidence regarding our practice and would enable us to implement any changes in an appropriate time frame, if required. We randomly selected cases for review using computer generated random numbers, identified clopidogrel-PPI co-administration and also established whether there was a strong indication for the PPI use as per National Institute for Health and Clinical Excellence (NICE) guidelines." We categorised patients into those who were on a PPI prior to admission and those started on a PPI during the admission process. For the former group, we considered appropriate indications for a PPI to be the following: any prior dyspepsia/ gastritis, gastroesophageal reflux, upper gastrointestinal (GI) bleed, Barrett's oesophagus, initiation following recommendation by a specialist (identified in the patient's GP summary or previous gastroenterology clinic visit at the hospital or in the past medical history). The use of PPI was considered inappropriate in cases where it was used in the absence of any $\mathrm{Gl}$ symptoms or to prevent possible drug-induced dyspepsia/gastritis. If the patients had any symptoms consistent with dyspepsia/gastritis, gastroesophageal reflux or signs of upper Gl bleed during admission, PPI was deemed appropriate. We considered the PPI use inappropriate if patients were started on it 'prophylactically' without sufficient justification.

Following completion of the audit, we agreed on local guidelines and implemented a simple algorithm (Figure I) which was provided to all junior doctors in a brief education session on their induction into the cardiology team. We also raised awareness among all the medical registrars who started in the hospital in August 2009 by delivering the same information. We prompted junior doctors to confirm the indication of PPI use before its initiation; if there was a genuine need, an appropriate one was encouraged (lansoprazole or pantoprazole rather than esomeprazole or omeprazole).

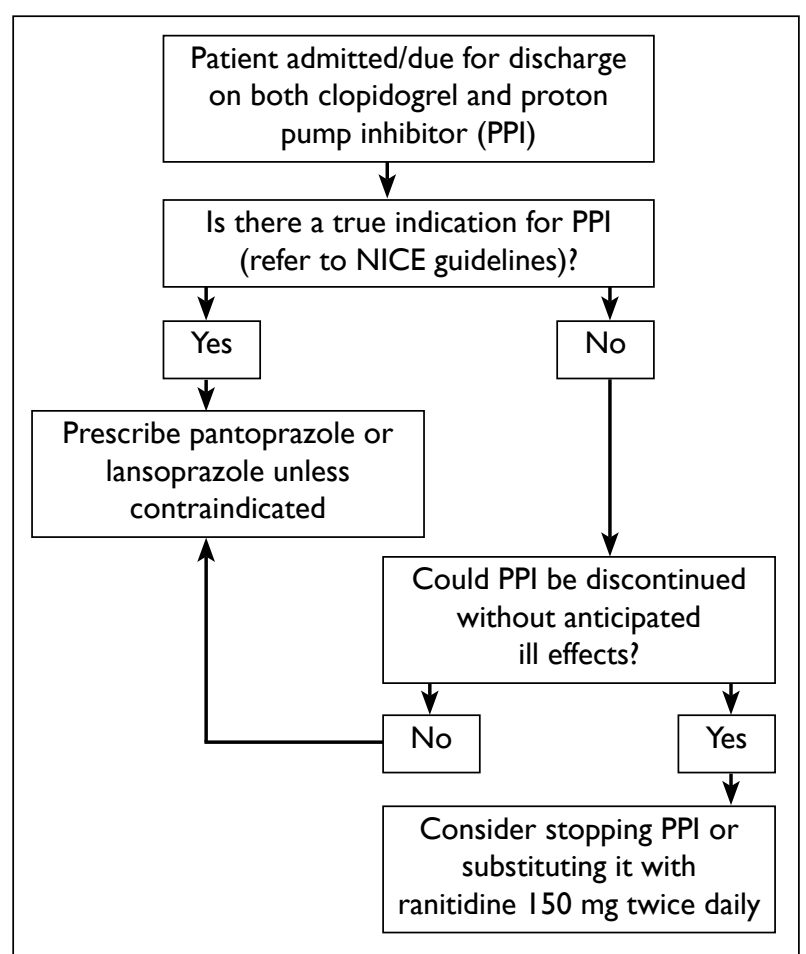

FIGURE I The simple algorithm we introduced summarising our guidance. This guidance was later supported by international recommendations from the European Medicines Agency. ${ }^{6}$

We audited all the patients discharged on clopidogrel following diagnosis of ACS in the period between August 2009 and February 2010. The same methods as the initial audit were used for the inclusion criteria and the categorisation of the patients into appropriate and inappropriate use of PPI.

Following the recommendations of the EMEA we asked other members of the multidisciplinary team (pharmacists and nurses in particular) to monitor all patients in the cardiac wards admitted with an ACS on a daily basis and to identify and flag-up patients on both clopidogrel and PPI so that the medical members of the team could assess whether this treatment was appropriate.

We measured the difference in correct use of PPI between the two periods that were audited, using the chi-squared

TABLE I The number of patients on clopidogrel following a diagnosis of acute coronary syndrome who were discharged on a proton pump inhibitor in the period between August 2008 and July 2009

\begin{tabular}{|l|l|}
\hline $\begin{array}{l}\text { Proton pump inhibitor used on } \\
\text { discharge pre-intervention }\end{array}$ & $\mathbf{n = 9 1}$ \\
\hline Omeprazole & $29(31.9 \%)$ \\
\hline Esomeprazole & $2(2.2 \%)$ \\
\hline Lansoprazole & $9(9.9 \%)$ \\
\hline Pantoprazole & $3(3.3 \%)$ \\
\hline Total & $\mathbf{4 3 ( 4 7 . 5 \% )}$ \\
\hline
\end{tabular}


TABLE 2 Clopidogrel-PPI co-administration following our intervention ( $\mathrm{n} / \mathrm{a}$ numbers are too small to calculate)

\begin{tabular}{|l|l|l|l|}
\hline $\begin{array}{l}\text { Proton } \\
\text { pump } \\
\text { inhibitor } \\
\text { prescribed }\end{array}$ & $\begin{array}{l}\text { Pre- } \\
\text { intervention } \\
(\mathbf{n = 9})\end{array}$ & $\begin{array}{l}\text { Post- } \\
\text { intervention } \\
(\mathbf{n}=101)\end{array}$ & $\begin{array}{l}\text { P-value } \\
\text { chi- } \\
\text { square } \\
\text { test }\end{array}$ \\
\hline Omeprazole & $29(31.9 \%)$ & $17(16.8 \%)$ & $\mathrm{p}=0.147$ \\
\hline Esomeprazole & $2(2.2 \%)$ & $3(3.0 \%)$ & $\mathrm{n} / \mathrm{a}$ \\
\hline Lansoprazole & $\mathbf{9 ( 9 . 9 \% )}$ & $4(3.9 \%)$ & $\mathrm{n} / \mathrm{a}$ \\
\hline Pantoprazole & $\mathbf{3 ( 3 . 3 \% )}$ & $3(3.0 \%)$ & $\mathrm{n} / \mathrm{a}$ \\
\hline Total & $\mathbf{4 3 ( 4 7 . 5 \% )}$ & $\mathbf{2 7}(\mathbf{2 6 . 7 \% )}$ & $\mathbf{p}=\mathbf{0 . 0 1 8}$ \\
\hline
\end{tabular}

test to assess statistical significance. P-values were twotailed and statistical significance was considered for $\mathrm{p}$-values $<0.05$. All calculations were performed using Intercooled Stata 8.2 (Stata Corp., College Park,TX).

\section{RESULTS}

In the period between August 2008 and July 2009, 253 patients were discharged on clopidogrel following a diagnosis of ACS. We randomly selected 100 cases for review using computer generated random numbers. We were able to identify 91 of these cases in time for our audit completion. Out of the 9I patients, 43 (47.5\%) were discharged on a PPI and more than two-thirds of those were on esomeprazole/omeprazole. The results of the 91 patients are shown in Table I. Of the 29 patients taking omeprazole only 9 (31\%) had an appropriate and documented indication for PPI use.

In the re-audit we performed following the change of our policy and the education session provided to junior doctors, we identified 128 patients and reviewed $10 \mathrm{I}$ case notes. There was a statistically significant decrease in the number of PPI prescriptions, driven predominantly by a decrease in the number of omeprazole prescriptions (Table 2).

\section{REFERENCES}

I Yusuf S, Zhao F, Mehta SR et al. Effects of clopidogrel in addition to aspirin in patients with acute coronary syndromes without ST-segment elevation. New EnglJ Med 200 I; 345:494-502. http://dx.doi.org/I0.1056/ NEJMoa0I0746

2 National Institute of Health and Clinical Excellence (NICE). Unstable angina and NSTEMI: the early management of unstable angina and non-ST-segment-elevation myocardial infarction [Internet] NICE; 2010 [cited 201I Oct 2I]. Available from: http://www.nice. org.uk/nicemedia/live//2949/47988/47988.pdf

3 National Institute of Health and Clinical Excellence (NICE).. Secondary prevention in primary and secondary care for patients following a myocardial infarction [Internet]. NICE; 2007 [cited 20I I Oct 2I]. Available from: http://www.nice.org.uk/nicemedia/ live/ I I008/30495/30495.pdf

4 CURRENT-OASIS 7 Investigators, Mehta SR, Bassand JP et al. Dose comparisons of clopidogrel and aspirin in acute coronary syndromes. New Engl J Med 2010; 363:930-42.

5 Juurlink DN, Gomes T, Ko DT et al. A population-based study of the drug interaction between proton pump inhibitors and clopidogrel. CMAJ 2009; I80:7I3-8. http://dx.doi.org/I0.I503/cmaj.08200 I
We also found that 13 out of 17 patients (77\%) now taking omeprazole had an appropriate and documented indication for PPI use.The use of ranitidine increased from $4 \%$ to $54 \%$ during this period. We believe that this increase is a result of a reduction in the use of PPI as a 'prophylactic' to reduce the risk of bleeding when patients took clopidogrel. When the risk of bleeding was deemed significant, ranitidine was being appropriately prescribed instead.

After the second audit we carried out four spot-checks once monthly over four consecutive months to ensure that the algorithm was firmly established as a standard procedure. We reviewed 17 patients and confirmed that no patient was discharged on omeprazole or esomeprazole when taking clopidogrel.

\section{DISCUSSION}

Co-administration of clopidogrel and PPI can be harmful because the efficacy of clopidogrel can be decreased. If omeprazole or esomeprazole are used, for example, there is concern about increased major cardiac events. There are therefore international guidelines from the EMEA and the FDA suggesting that PPI should not be used unless indicated and in this case pantoprazole or lansoprazole are preferable. Our audit and the simple algorithm we created following its findings resulted in a significant reduction in the co-administration of a PPI and clopidogrel. At the same time we increased the appropriate use of PPI, demonstrating the effectiveness of our intervention. The co-administration of ranitidine and clopidogrel is considered a safer alternative and prescription numbers have correspondingly increased. We also showed that the resulting changes were sustained among our multidisciplinary teams over time. We feel that the simplicity of the algorithm and the use of the multidisciplinary team were instrumental to the success of this audit. We recommend that similar simple measures could be taken in most hospitals to address similar issues.

6 European Medicines Agency. Public statement interaction between clopidogrel and proton-pump inhibitors [Internet]. London: EMA; 2010 [cited $201 \mathrm{I}$ Oct 2I]. Available from: http://www.ema.europa.eu/docs/en_GB/document_ library/Public_statement/2010/03/WC500076346.pdf

7 Bhatt MD, Cryer BL, Contant FC et al. Clopidogrel with or without omeprazole in coronary artery disease. N EnglJ Med 2010;363:1909_ I7. http://dx.doi.org/I0.I056/NEJMoal007964

8 Southworth MR, Temple R. Interaction of clopidogrel and omeprazole. N Engl J Med 2010; 363:1909-17. http://dx.doi. org/I0.I056/NEJMcI0I2022

9 US Food and Drug Administration (FDA). Reminder to avoid concominant use of plavix (clopidogrel) and omeprazole [Internet]. Maryland: FDA; 2010 [cited 2012 Jan I5]. Available from: http:// www.fda.gov/Drugs/DrugSafety/ucm23 I I6I.htm

10 Kimmey MB, Silverstein FE. Role of $\mathrm{H} 2$-receptor blockers in the prevention of gastric injury resulting from nonsteroidal antiinflammatory agents. Am J Med 1988; 84:49-52. http://dx.doi. org/10.1016/0002-9343(88)90254-9

II National Institute of Health and Clinical Excellence (NICE). Dyspepsia: managing dyspepsia in adults in primary care [Internet]. NICE; 2004 [cited 20I I Oct 2I]. Available from: http://www.nice. org.uk/nicemedia/live/ I0950/29459/29459.pdf 